\title{
Apuntes para una biografía política de Rafael Gasset, un liberal regeneracionista
}

\author{
Gregoria Villanueva Larraya *
}

Rafael Gasset desde su posición privilegiada de director del prestigioso diario El Imparcial, propiedad de la familia, comenzaba en abril de 1899 una campaña de propaganda ${ }^{1}$ a favor de la reconstrucción económica del país centrada en el fomento de las obras públicas y muy en particular de las obras hidráulicas con destino al riego, con la finalidad de aumentar la producción agraria y la riqueza de la nación. Era la señal de su incorporación al movimiento regeneracionista y el inicio de un trabajo político encaminado a poner en práctica buena parte de las aspiraciones expuestas desde los años ochenta por Joaquín Costa, y que a raíz de la pérdida de las colonias habían tenido una buena acogida en amplios sectores de opinión.

Las ideas de reconstrucción interior o "reconstitución nacional», según la terminología de la época, junto a la de «regeneración» y «europeización" fueron muy pronto asimiladas, como es bien sabido, por los políticos del turno. En el caso de la reconstrucción económica, que es la que nos interesa destacar aquí los problemas surgieron de inmediato a la hora de arbitrar los recursos imprescindibles para llevarla a término, dada la permanente debilidad de la Hacienda Pública. El escaso margen de actuación que dejaba el presupuesto ordinario para invertir en gastos

* UNED.

"El Imparcial: "Para la nueva política. Los canales de riego». 7 de abril a 7 de junio de 1899. 
"reproductivos», es decir, generadores de riqueza obligaba a solicitar de las Cortes la aprobación de créditos extraordinarios.

La singularidad de Rafael Gasset como personalidad política radica precisamente en que adscribió su tarea de gobierno a conseguir un presupuesto extraordinario para el Ministerio de Fomento con la finalidad de comenzar un período constructivo extraordinario de obras públicas, que valoraba «salvador» para los intereses de España, por cuanto permitiría la reactivación económica de la nación y la obtención a medio y largo plazo de nuevos recursos para el Tesoro mediante el incremento de la riqueza imponible.

Los planes de Gasset no lograron el necesario acuerdo parlamentario, por lo que los resultados conseguidos a la llegada de Primo de Rivera distaban mucho de los ambiciosos objetivos propuestos a primeros de siglo.

La falta de éxito de su gestión se debió a una suma combinada de causas, entre las que cabe señalar: la situación crítica de la Hacienda pública a raíz de la guerra colonial; la aceptación de la política de nivelación presupuestaria más allá de las previsiones del propio Villaverde; la reaparición del déficit público en 1909 y el aumento considerable del mismo a partir de 1912, debido en gran parte a la acción en Marruecos; la persistente debilidad de los ingresos de la Hacienda pública para hacer frente a gastos crecientes; las luchas de partido que impedian acuerdos parlamentarios; la disolución de los partidos dinásticos; la negativa de los núcleos de poder económico que sustentaban el sistema de la Restauración a ceder un mínimo de sus privilegios.

\section{RAFAEL GASSET EN EL GOBIERNO DE LA NACIÓN. SU PROGRAMA}

En abril de 1900 Rafael Gasset era llamado por Silvela para ocupar la cartera de Agricultura. No es extraño que el presidente del Gobierno, en su deseo de incorporar y asimilar las propuestas regeneracionistas, acudiera a quien había defendido un programa de «reconstitución», que conectaba con importantes sectores de población partidarios de salir de la crisis del 98 mediante el desarrollo progresivo de la riqueza material del país, y que contaba, además, con el apoyo de uno de los periódicos de mayor difusión. 
Rafael Gasset en su tarea parlamentaria ${ }^{2}$ y en su campaña periodística había defendido «una labor de Gobierno armónica y coherente», fundamentada en tres pilares: la enseñanza, el fomento de la riqueza y la defensa de la nación, pero mostrándose, al igual que Joaquín Costa, más favorable a «atender con primordial cuidado la escuela y el campo", ya que, afirmaba, "España para volver a ser fuerte, necesita primero ser rica».

Siendo la agricultura todavía la principal fuente de riqueza del país, Gasset estimó que la acción del Estado ${ }^{3}$ debía dirigirse a aumentar la exigua producción agrícola por el sistema a su juicio más eficaz: el aprovechamiento y utilización de las aguas de dominio público con destino al riego. La extensión del regadío hasta el máximo de las posiblidades naturales debía contribuir a la solución de la "cuestión agraria». En consecuencia, su nombramiento como ministro de Agricultura abría la posibilidad de poner en práctica la "política hidráulica" costiana y renovaba la preocupación hidraulista habida en los Gobiernos liberales desde mediados del siglo XIX.

Desde un principio, y continuando las orientaciones de Costa, quiso dar al programa de "reconstitución nacional» el rango de programa nacional, al margen de tendencias partidistas: "Sean las que fueren las opiniones políticas de cada uno, trabajamos por España, que está por encima de los criterios, de las escuelas, de las ideas, de los partidos” ${ }^{4}$.

Ocupó el cargo de ministro de Agricultura, Industria y Comercio (llamado otra vez de Fomento a partir de 1905) en nueve ocasiones ${ }^{5}$. En un principio con los Gobiernos conservadores de Silvela y Villaverde, y a la muerte de este con Gobiernos del Partido Liberal, al que se había incorporado por mediación de Segismundo Moret, antiguo defensor de la intervención estatal en las grandes obras hidráulicas e introductor de la

${ }^{2}$ Diario de Sesiones del Congreso, núm. 33, 11 de julio de 1899, págs. 821-824. Proposición presentada por Rafael Gasset solicitando del Gobierno una declaración en la que éste mostrara su disposición "a tomar a su cargo como función propia del Estado la construcción de canales y pantanos de riego ...".

${ }^{3}$ Exposición al Real Decreto sobre reorganización del servicio hidráulico, 11 de mayo de 1900. 75.

${ }^{4}$ "Mitin de Rafael Gasset en Alicante», Revista de Obras Públicas. Madrid (1902), pág.

${ }^{5}$ Gobierno de Silvela, abril-octubre 1900. Gobierno Villaverde, julio-diciembre 1903. Gobierno Moret, diciembre 1905-julio 1906; noviembre-diciembre 1906; octubre 1909-febrero 1910. Gobierno Canalejas, marzo 1911-marzo 1912. Gobierno Romanones, marzo-octubre 1913; abril 1916-abril 1917. Gobierno Concentración Liberal, diciembre 1922-septiembre 1923. 
política hidráulica en el Gobierno del Partido Liberal, sin duda inducido por la sequía y la consiguiente crisis agraria de 1904-1905.

Los escasos períodos de tiempo en el desempeño de sus funciones de gobierno, fiel reflejo de la inestabilidad política de la época, no fueron obstáculo para que defendiera apasionada e insistentemente ${ }^{6}$ su programa de «reconstitución» mediante un período constructivo extraordinario de obras públicas y en particular de obras hidráulicas, al que quiso dedicar por entero su actividad política y de gobierno, de tal modo que no aceptó ningún cargo público que no fuera el de ministro de Fomento.

\section{EL PLAN DE OBRAS HIDRÁULICAS DE 1902}

El logro más importante de su primera estancia en el Ministerio de Agricultura (abril a octubre de 1900) fue la reorganización del servicio hidrológico, con el fin de elaborar en el plazo de un año un plan general de obras hidráulicas. Este plan fue aprobado con carácter provisional por el Real Decreto de 25 de abril de 1902, durante el Gobierno de Sagasta, siendo José Canalejas ministro de Agricultura. Proyectaba regar cerca de 1.470 .000 hectáreas $^{7}$ gracias a la construcción de 296 obras entre pantanos y canales.

El plan aprobado resultó ser un amplio catálogo de obras a construir, pendientes de estudios técnicos, al que no se adjuntaba ninguna consignación presupuestaria, ni normas para su ejecución, ni orientaciones agronómicas ni económicas.

A pesar de sus deficiencias, el Plan de 1902 se convirtió en el punto de referencia obligado para la elaboración de los posteriores planes de obras hidráulicas de 1909, 1916 y 1919, más restringidos y coyunturales, que pretendian beneficiar a $330.000,570.000$ y 590.000 hectáreas respectivamente ${ }^{8}$.

- Su tenacidad para defender la política hidráulica fue en ocasiones ridiculizada: se le llamaba «Miangairriéguez".

${ }^{7}$ La superficie regable se distribuía por Divisiones hidrológicas de la siguiente manera: Pirineo Oriental $30.000 \mathrm{Ha}$; Ebro 327.000; Júcar 143.000; Segura 14.300; Sur de España 9.400; Guadalquivir 177.900; Guadiana 406.602; Tajo 181.850; Duero 152.600; Miño y Cantabria $25.900 \mathrm{Ha}$.

${ }^{8}$ Planes de obras hidráulicas que formaban parte de planes más amplios de obras públicas incluidos en proyectos de petición de créditos extraordinarios, solicitados por el Ministerio de Fomento para impulsar la reconstrucción del país. Ninguno de ellos obtuvo la aprobación de las Cortes. 
La aprobación del Plan de obras hidráulicas, que debía ser en gran parte construido a cargo del Estado, obligaba a plantear el modo de armonizar dos políticas aparentemente incompatibles: Por un lado la política de nivelación presupuestaria, que exigía importantes sacrificios a las inversiones estatales, y por otro el carácter expansionista de un programa de desarrollo económico como el que Gasset representaba. La política de nivelación presupuestaria, aunque había sido criticada por las Cámaras Agrícolas y de Comercio, fue en general aceptada tanto por el Partido Conservador como por el Liberal, lo que permitió, más allá de las propias previsiones de Fernández Villaverde, saldar con superávit los presupuestos de los primeros ocho años del siglo ${ }^{9}$.

Al incorporarse al Gobierno Silvela, Gasset aceptó la política de nivelación, pero muy pronto pidió de igual modo un esfuerzo para llevar adelante una política mínimamente inversora ${ }^{10}$ que impulsara el desarrollo de la riqueza pública, si no se quería aumentar todavía más la distancia que separaba a España de los países más adelantados de Europa. Se continuaba hablando de regeneración, pero la realidad era que los distintos Gobiernos oponían una constante y desconsoladora resistencia pasiva a los impulsos para desarrollar la infraestructura del país. Los presupuestos eran cada año una repetición del anterior, y se dejaba para el siguiente la tarea de modificarlos.

\section{PLANES DE OBRAS PÚBLICAS Y PETICIÓN DE CRÉDITO EXTRAORDINARIO}

El 9 de marzo de 1911 Rafael Gasset, ministro de Fomento del Gobierno presidido por Canalejas, presentaba en las Cortes varios proyectos de ley de obras públicas ${ }^{11}$ dirigiéndose a los diputados con estas

${ }^{9}$ Solé Villalonga, Gabriel, La reforma fiscal de Villaverde. Madrid, 1967, págs. 264.

${ }^{10}$ Diario de Sesiones del Congreso, núm. 100, 17 de diciembre de 1903, págs. 3.1223.125; núm. 43, 11 de diciembre de 1907, págs. 950-954. «Los riegos en Egipto. Conferencias en el Ateneo de Madrid», Revista de Obras Públicas. Madrid (1905), págs. 329-330; 351-353; 359.

${ }^{11}$ Diario de Sesiones del Congreso, Apéndices 1 a 8, núm. 4, 9 de marzo de 1911. Proyectos de ley "suprimiendo el plan de carreteras del Estado"; "sobre caminos vecinales»; «sobre construcciones hidráulicas»; «sobre reorganización e inspección de las Juntas de obras de puertos"; "reforma de la ley de ferrocarriles secundarios"; "reforma de la ley de expropiación forzosa»; "sobre servicios de agricultura»; "sobre repoblación forestal». 
palabras: «Os aseguro que no lo hago sin cierta emoción, porque en esta obra he puesto diez años de mi vida, diez años de trabajo, he puesto algo más: la ilusión de poder unir mi nombre a una obra de reconstitución de mi Patria».

El Gobierno Canalejas parecía estar decidido a emprender un período constructivo extraordinario y dar así respuesta a las peticiones hechas desde la pérdida de las colonias por los centros productores y mercantiles. Los proyectos de obras elaborados bajo la dirección de Gasset tenían como objetivos inmediatos ${ }^{12}$ promover el trabajo nacional y detener la fuerte emigración habida durante el primer decenio del siglo, para lo cual se solicitaba de las Cortes la aprobación de un crédito extraordinario de 320 millones para cinco años, porque no era posible acometer tal empresa con los escasos recursos que proporcionaba el presupuesto ordinario.

Ese esfuerzo constructivo debia estar presidido por un principio de justicia distributiva entre todas las regiones, que dejando de lado la mayor o menor influencia de los representantes en Cortes basara los proyectos en estudios técnicos para garantizar un máximo de rendimiento de las obras. Gasset proponía modificar ${ }^{13}$ la orientación de las inversiones en obras públicas realizadas hasta entonces por el Ministerio de Fomento, que en los últimos 50 años había dedicado la mayor parte de su presupuesto a construir un vasto plan de carreteras. Como consecuencia España contaba con $44.000 \mathrm{~km}$ de carreteras nacionales construidas $^{14}, 5.000 \mathrm{~km}$ más que Francia (país con un nivel económico muy superior), y tenía vigente un plan que proyectaba construir $40.000 \mathrm{~km}$ más, plan que no había nacido de unos proyectos técnicos sino de la yuxtaposición de las proposiciones hechas por distintos diputados. Llegaba por tanto la hora de poner fin a la llamada "carretera parlamentaria», criticada años ha por Costa.

Para ello los proyectos de Gasset iban dirigidos en primer lugar a suprimir el vigente plan de carreteras, respetando de él la construcción de sólo $7.000 \mathrm{~km}$, y a prestar un gran apoyo a la construcción de caminos vecinales, cuya diferencia con el país vecino resultaba abrumadora: $20.000 \mathrm{~km}$ frente a $600.000 \mathrm{~km}^{15}$. La construcción de caminos vecinales

${ }^{12}$ Diario de Sesiones del Congreso, núm. 4, 9 de marzo de 1911, págs. 38-39.

${ }^{13}$ Ibidem, págs. 36-37.

${ }^{14}$ Diario de Sesiones del Congreso, "Exposición al proyecto de ley suprimiendo el plan de carreteras del Estado", Apéndice 1. ${ }^{\circ}$ al núm. 4, 9 de marzo de 1911.

${ }^{15}$ Diario de Sesiones del Congreso, «Exposición al proyecto de ley sobre construcción de caminos vecinales", Apéndice $3 .^{\circ}$ al núm. 4, 9 de marzo de 1911. 
había de hacerse, el igual que ya se había realizado en otros países europeos, mediante un esfuerzo conjunto del gobierno central con las provincias y municipios. El nuevo proyecto de ley concedía subvenciones y anticipos para la construcción a los pueblos y comarcas, aun cuando Gasset fuera consciente de las dificultades del proyecto en la práctica por la debilidad, e incluso en algunos casos inexistencia de las haciendas locales.

En la construcción de puertos ${ }^{16}$ había sucedido algo parecido a lo ocurrido con las carreteras. Mientras los pueblos más ricos de Europa habían concentrado sus esfuerzos en construir y acondicionar seis $u$ ocho puertos, en España, aun cuando la ley de Puertos de 7 de mayo de 1880 había fijado el número de puertos de interés general en 28 , éstos habían alcanzado la cifra de 132 en virtud de la aprobación de leyes especiales. El proyecto de ley presentado no quiso remover los intereses creados en torno a la construcción de los nuevos puertos aprobados, sino que se limitó a exigir mayores requisitos para la organización de las Juntas de Obras de puertos y a destinar 60 millones del crédito extraordinario solicitado para agilizar la construcción y subvencionar a nueve puertos (Barcelona, Valencia, Algeciras, Sevilla, Santander, Bilbao, La Luz, Ceuta y Melilla) por entender que éstos eran los de mayor importancia comercial y política.

El proyecto de ley sobre construcciones hidráulicas ${ }^{17}$ por un lado pretendía intensificar el ritmo de construcción del plan de obras hidráulicas por el que se solicitaban 105 millones de pesetas, y por otro regulaba los procedimientos para la ejecución de las grandes obras hidráulicas. Éstas podrían ser ejecutadas: 1. Por el Estado con el auxilio de las localidades interesadas. 2. Por Asociaciones o Empresas con el auxilio del Estado. 3. ${ }^{\circ}$ Por cuenta exclusiva del Estado, siempre y cuando se hubiera demostrado la imposibilidad de los otros procedimientos. La intervención exclusiva del Estado continuaba planteándose como una acción subsidiaria de la iniciativa privada ${ }^{18}$, pero a sabiendas, como afirmaba Gasset, de que «el Estado tendrá que suplir(la) en los más de

${ }^{16}$ Diario de Sesiones del Congreso, "Exposición al proyecto de ley sobre reorganización e inspección de las Juntas de Obras de Puertos", Apéndice 4 al núm. 4, 9 de marzo de 1911.

${ }_{17}$ Diario de Sesiones del Congreso, «Exposición al proyecto de ley sobre construcciones hidráulicas", Apéndice 2 al núm. 4, 9 de marzo de 1911.

${ }_{18}$ Opinión mantenida por Ortega Cantero, Nicolás, Politica agraria y dominación del espacio. Madrid, Ed. Ayuso, 1979, pág. 51. 
los casos" ${ }^{19}$. De cualquier modo, a partir de la aprobación de la ley los obstáculos para la construcción de las grandes obras hidráulicas no derivaron tanto de un problema doctrinal sobre la intervención o no del Estado en dichas obras, que estaba prácticamente superado, sino de un problema de asignación de recursos al Ministerio de Fomento, o sea, de la voluntad política de llevar aquéllas a cabo.

Los proyectos de ley sobre caminos vecinales y construcciones hidráulicas, que eran los de mayor interés para Gasset, fueron aprobados sin grandes dificultades en lo referente a la regulación del procedimiento administrativo para construir las obras. Los problemas se presentaron a la hora de decidir la oportunidad o no de dotar al Ministerio de Fomento con el crédito que solicitaba. El Partido Conservador, alarmado por el aumento del gasto público $-\mathrm{y}$ por lo tanto del déficit- registrado a partir de 1909, y enfrentado al Gobierno Canalejas, entre otros motivos por el proyecto de supresión del impuesto de consumos, anunció muy pronto su rechazo a tal solicitud ${ }^{20}$.

Gasset calificó de «labor perturbadora» ${ }^{21}$ la decisión de la minoría conservadora de retrasar indefinidamente la aprobación de unos créditos encaminados a «promover el trabajo nacional». Defendía, por el contrario, que cuanto más difíciles fueran las circunstancias de la Hacienda pública, mayor obligación había de acudir a los gastos de desarrollo. Retrasar ${ }^{22}$ la política de "reconstitución" interior provocaba no sólo una distancia cada vez mayor con los países más progresivos de Europa, sino también una desconfianza del electorado respecto a la clase política por los continuos ofrecimientos hechos, pero siempre incumplidos.

Se quejaba, al igual que lo habían hecho otros regeneracionistas, de la proclividad de los gobiernos para dotar de recursos a las aventuras externas mientras se descuidaba el desarrollo de la riqueza interna. Así, ante la firma del tratado franco-español sobre Marruecos (27 de noviembre de 1912) y las obligaciones a que comprometía el mismo, Gasset no dudó en proclamar la necesidad de simultanear ${ }^{23}$ la empresa africana con la política de reconstrucción interior, buscando ante todo la "proporcionalidad en los gastos». Resumía su pensamiento en la siguiente frase:

${ }^{19}$ Diario de Sesiones del Congreso, núm. 4, 9 de marzo de 1911, pág. 36.

${ }^{20}$ Diario de Sesiones del Senado, rúm. 54,9 de junio de 1911, págs. 799-804, 808809; núm. 55, 10 de junio de 1911, págs. 812-814, 818-819. Intervención de Allendesalazar.

${ }^{21}$ lbidem, pág. 805.

22 Ibidem, págs. 807-808, 817.

${ }^{23}$ Primer Congreso Nacional de Riegos. "Sesión inaugural». Discurso de Rafael Gasset, pág. 58. Zaragoza, 1913. 
«No para España lo que sobre de Marruecos, sino para Marruecos lo que sobre de España.»

La oposición del Partido Conservador a las propuestas económicas de Rafael Gasset estuvo acompañada de una fuerte crítica al desarrollo y resultados de la «política hidráulica» ${ }^{24}$ y en general hacia su gestión en el Ministerio de Fomento, del que dimitía en marzo de 1912 al ser acusado por la minoría conservadora ${ }^{25}$ en el Congreso de introducir modificaciones al reciente plan de carreteras.

\section{PRESUPUESTO DE RECONSTITUCIÓN NACIONAL DE SANTIAGO ALBA Y PLAN DE OBRAS PÚBLICAS DE RAFAEL GASSET}

En la primavera de 1916 Rafael Gasset accedía de nuevo al Ministerio de Fomento, formando parte del gobierno presidido por Romanones y cuyo hombre fuerte era el ministro de Hacienda, Santiago Alba. La situación económica y social del pais era difícil (notable aumento del déficit público, alta tasa de inflación, problemas de subsistencias, descontento en el Ejército y en la Administración Civil, problemas laborales y de orden público), pero ello no impidió sino que impulsó a Alba a plasmar sus ideas regeneracionistas en medidas de política económica ${ }^{26}$ encaminadas a simultanear la política de nivelación y la reconstitución como un imperativo que la realidad imponía. Por otra parte, la Guerra Europea y la adopción de una postura de neutralidad por parte del Gobierno ante los países beligerantes abría, en opinión de Gasset, un lapso de tiempo excepcional, que debía ser aprovechado por la nación para reducir el secular atraso respecto a Europa: «O utilizamos este plazo para unirnos a Europa, o quedaremos por siempre enormemente distanciados de ella...” ${ }^{27}$.

${ }^{24}$ Oposición protagonizada muy en especial por Allendesalazar, ex-ministro maurista y portavoz de la minoría conservadora en el Senado de 1911 a 1913.

${ }^{25}$ Diario de Sesiones del Congreso, n. ${ }^{\circ} 98,2$ de marzo de $1912 ; n .{ }^{\circ} 99,4$ de marzo de 1912; $\mathrm{n}^{\circ}$ 101, 6 de marzo de 1912.

${ }^{26}$ GARCía LOPEZ, José, «El programa económico y financiero de Alba». Papeles de Economía Española, n. ${ }^{\circ} 20$. Madrid, (1984), págs. 216-235.

${ }_{27}$ GASSET, Rafael, Reforma de los Presupuestos. El Plan extraordinario de Obras Públicas. Madrid, 1916, pág. 9. 
El programa económico y financiero de Alba proponía en primer lugar reducir el gasto público mediante un recorte de presupuestos, en particular de los gastos dedicados a la acción en Marruecos; en segundo lugar, intensificar los ingresos del Tesoro mediante la modificación de alguno de los impuestos vigentes y el establecimiento de otros nuevos, comenzando por el impuesto sobre beneficios extraordinarios de las sociedades y particulares a causa de la Guerra; en tercer lugar, responder a a las necesidades más urgentes de la reconstitución nacional mediante una política financiera ajustada.

Del conjunto de proyectos de ley presentados por Alba en el Congreso, el Presupuesto de Reconstitución Nacional ${ }^{28}$, cifrado en $2.100 \mathrm{mi}$ llones de pesetas para diez años, es la pieza central de su programa económico. Atendía en primer lugar a la consolidación de la Deuda flotante del Tesoro y en segundo, a la ejecución de un Plan extraordinario de obras públicas elaborado bajo la dirección de Gasset desde el Ministerio de Fomento, y cuyo presupuesto ascendía a 1.073 millones de pesetas para diez años.

El Plan extraordinario de obras públicas, si bien no suponía un «plan integral» de reconstitución nacional, imposible de plantear, según Gasset, no sólo por la escasez de recursos materiales sino también por la falta de estudios técnicos en algunos campos, pretendía al menos realizar «un primer gran esfuerzo constructivo" para responder a necesidades que no admitían demora ${ }^{29}$. Sus objetivos, continuación de lo proyectado en 19 i 1 , quedaban contenidos en dos grandes apartados: Primero, atención a las vías de comunicación, dando prioridad a la reparación de la red de carreteras y a la construcción de caminos vecinales, puentes y puertos, y dejando al margen del presupuesto extraordinario la construcción de ferrocarriles secundarios. Segundo, el estímulo de la producción del país favoreciendo el desarrollo de la riqueza agrícola, en especial por la construcción de pantanos y canales de riego, por la repoblación forestal y la enseñanza agrícola.

Las minorías parlamentarias, a excepción de los radicales de Lerroux ${ }^{30}$, argumentaron, al igual que to habían hecho con el proyecto de Reconstitución Nacional, que el Plan de obras públicas era una «obra

${ }^{28}$ El artículo de García López contiene un estudio de la discusión en el Congreso del «Presupuesto de Reconstitución», págs. 225-231.

${ }^{29}$ Diario de Sesiones del Congreso, $n{ }^{\circ} 89,14$ de noviembre de 1916, págs. 27692771.

${ }^{30}$ Diario de Sesiones del Congreso, n. ${ }^{\circ} 94,20$ de noviembre de 1916, págs. 29532955. 
modesta», que no correspondía calificar de «reconstitución» porque dejaba fuera del proyecto un aspecto tan fundamental como el de los ferrocarriles, lo cual significaba no dar solución al problema de los transportes y de la circulación de la riqueza ${ }^{31}$. El debate parlamentario estuvo muy influenciado por la actitud que las minorías conservadora y regionalista habían adoptado ante el plan económico y financiero de Alba, y muy particularmente, como es bien sabido, ante el proyecto de ley sobre "beneficios extraordinarios". Una polémica estéril sobre qué debía aprobarse primero en la Cámara, si el Presupuesto ordinario para 1917 o el Presupuesto para la Reconstitución Nacional, es una buena demostración de la labor obstrucionista de las minorías, que se vio favorecida por la debilidad del Partido Liberal y la falta de acuerdo entre los diputados liberales respecto a los proyectos de Alba.

La oposición a aprobar los gastos extraordinarios supuso un nuevo freno para los planes constructivos de Rafael Gasset y la pérdida de una ocasión excepcionalmente favorable para reorganizar parte de la economía del país.

\section{7-1922. ALEJAMIENTO DE LA POLITICA DE PARTIDO Y DE LA ACCIÓN DE GOBIEFNO}

A raíz de una importante crisis por la que atravesó El Imparcial en $1917^{32}$, Rafael Gasset decidió permanecer al margen de toda actuación partidista y canalizar su esfuerzo personal en favor del programa de reconstitución nacional a través de la prensa, de las tareas parlamentarias y de conferencias y mítines.

Ante los nuevos problemas políticos planteados en esos años fue partidario de una "colaboración con las izquierdas" para llevar a cabo la reforma tributaria imprescindible y poner en práctica los planes de desarrollo económico y social, que más que nunca en su opinión no admitían

31 Ibidem, págs. 2945-2950.

32 Desvols, Jean Michel, La prensa en España (1900-1931). Madrid, Ed. Siglo XXI, 1977, págs. 12-14, 20-23, 53-55.

El Imparcial: "La propiedad y la dirección de El Imparcial. Unas cuartillas de Rafael Gasset". 18 de junio de 1917. 
demora, y a los que debían estar supeditadas las ambiciones de los nacionalistas y las peticiones de reforma constitucional ${ }^{33}$.

A partir de las elecciones de febrero de 1918 intentó aunar en torno a su persona y a su programa un núcieo de opinión liberal agrario que actuara dentro y fuera del Parlamento para exigir de los gobiernos una atención preferente hacia los problemas de «reconstitución nacional», y muy concretamente en defensa del afianzamiento de la agricultura. Sobre la base de una muy modesta representación parlamentaria - de cinco a ocho diputados y uno a tres senadores en las sucesivas elecciones de 1918, 19, 20 y 23- constituyó un núcleo parlamentario que defendió los intereses agrarios ${ }^{34}$, en particular los de los cerealistas que, disconformes con la tasa del trigo, la carestía de los abonos y la actuación de los sindicatos harineros, pedían al poder público la adopción de medidas urgentes no para subir el precio del pan sino para reducirlo y abaratar de igual modo la producción triguera.

Aludió en más de una ocasión a la posible formación de una Liga a favor de la reconstitución nacional que tuviera como cometido "vigilar la necesaria proporcionalidad en la aplicación de los recursos a los graves problemas que han de hacer culta, próspera y fuerte a la nación ${ }^{35}$, e incluso a la necesidad de organizar un gran partido agrario. Ninguna de las dos opciones cristalizó. Quizá la acción que tuvo más resonancia fue el acuerdo del grupo parlamentario de solicitar la adhesión de todas las Sociedades, Instituciones, Federaciones y Sindicatos de carácter agrario, que dio como resultado el envío al Congreso de los Diputados por dichas agrupaciones de telegramas solicitando del Parlamento una mayor atención y dedicación a los problemas de reconstitución económica.

\section{LA PARTICIPACIÓN EN EL GOBIERNO DE CONCENTRACIÓN LIBERAL (DICIEMBRE 1922-SEPTIEMBRE 1923).}

Gasset participó como ministro de Fomento en el gobierno de concentración liberal presidido por García Prieto con la esperanza de ver

${ }^{33}$ El Imparcial, "Mi actitud política", 10 de noviembre de 1917. "Ante el gran problema. Necesidad de un empréstito", 24 de julio de 1918.

${ }^{34}$ El Imparcial, "Defensa de los intereses agrarios», 25 de enero de 1919. «Importante reunión de senadores y diputados de comarcas trigueras», 5 de febrero de 1919.

${ }_{35}$ El Imparcial: "Segundo Congreso de Riegos. Discurso de. Rafael Gasset, 6 de mayo de 1918. 
finalmente aprobado un presupuesto extraordinario para la reconstitución nacional, tal y como se había anunciado en el programa de la concentración, de marcado carácter reformista. Alejándose de las cifras fantásticas propuestas por de La Cierva en $1921^{36}$, Gasset rebajaba sus aspiraciones a un presupuesto más realista de 1.500 millones de pesetas. No obstante, para disponer de esos recursos era preciso que el Gobierno acordara restricciones en los gastos para la acción en Marruecos ${ }^{37}$.

El programa de concentración liberal había propuesto liquidar lo antes posible las operaciones militares en Marruecos e implantar a continuación una política civilista limitada a cumplir el Tratado de 1912. Sin embargo, aunque en un principio el Gobierno rechazó una propuesta del General Martínez Anido sobre un posible desembarco en Alhucemas, el presidente García Prieto y el ministro de Estado, Alba, entre otros miembros del Consejo de ministros, aceptaron a finales del mes de agosto un informe emitido por el Estado Mayor Central que recomendaba intensificar las acciones militares y desestimaba a su vez la propuesta hecha por el Gobierno de establecer una nueva línea estratégica de segura y fácil defensa, que permitiría reducir los gastos de 600 a 200 millones de pesetas.

El 1 de septiembre de Rafael Gasset dimitía del Gobierno, acompañado por los ministros de Trabajo, Chapaprieta, y de Hacienda, Villanueva. A los pocos días explicaba ampliamente en El Imparcial las razones de su dimisión ${ }^{38}$. Acatar el informe del Estado Mayor suponía en su opinión aumentar ios recursos económicos a dedicar para Marruecos y un nuevo aplazamiento para comenzar la empresa de reconstrucción interior. «Siempre hay un requerimiento apremiante para emplear más y más en Marruecos; siempre una dilación, una demora, para lo que España razonada y clamorosamente demanda». Era hora ya de «realidades inmediatas" y no de "declaraciones de Gobierno".

El 13 de septiembre el general Primo de Rivera tomaba el poder. Rafael Gasset desde El Imparcial ${ }^{39}$ condenaba la sedición. Reafirmaba su fe en un poder civil y en la monarquía parlamentaria, pero no se asombraba de que "con cierto berieplácito de una parte de la opinión se pretend(iera) acabar con la esterilidad de un sistema político».

${ }^{36}$ Diario de Sesiones del Congreso, «Proyecto de ley relativo a Transportes, Obras Públicas y Fomento de la Riqueza Nacional», Apéndice $1 .^{\circ}$ al núm. 92,13 de mayo de 1921. Cifraba el presupuesto en 12.500 millones de pesetas para diez años.

${ }^{37}$ Diario de Sesiones del Congreso, núm. 28, 13 de julio de 1923, págs. 941-943.

${ }^{38}$ El Imparcial, «Justificando una actitud. La reconstitución nacional», 7 de septiembre de 1923.

${ }^{39}$ El Impacial, «No es lícito callar. En las horas críticas", 28 de septiembre de 1923. 